\title{
Computation of Latent Heat based on the Energy Distribution Histogram in the 3D Ashkin-Teller Model
}

\author{
D. Jeziorek-Kniola*, Z. Wojtkowiak and G. Musiat \\ Faculty of Physics, A. Mickiewicz University, Umultowska 85, 61-614 Poznań, Poland
}

\begin{abstract}
The method of computation of the latent heat based on the energy distribution histogram is applied to the standard 3D Ashkin-Teller (AT) model. Similarly as in the original method for the $q$-state Potts model for strong first order phase transitions, the characteristic histogram with two peaks in the critical region have been observed. Positions of two minima of negative logarithm of internal energy probability for samples of finite size show good linear scalability to the thermodynamic limit. The applicability of this method has been confirmed by proving that the latent heat values are consistent with the ones obtained by us using the analysis of the behavior of the cumulants of the type of Challa and of Lee-Kosterlitz. The presented method is far more efficient than the one based on those cumulants.
\end{abstract}

DOI: 10.12693/APhysPolA.133.435

PACS/topics: $75.10 . \mathrm{Hk}, 75.30 . \mathrm{Kz}, 75.40 . \mathrm{Mg}$

\section{The standard Ashkin-Teller model}

The Ashkin-Teller (AT) model originally proposed for four component mixture [1], but later was expressed by Fan [2] in terms of two Ising models put on the same lattice with spins $s_{i}$ and $\sigma_{i}$ at each lattice site $i$. In consequence, only two-spin interactions of a constant magnitude $J_{2}$ between the nearest neighbors are considered. These Ising models are extended to the AT one by the four-spin interaction of a constant magnitude $J_{4}$ also only between couples of nearest-neighboring spins. Thus, the effective Hamiltonian $H$ is of the form

$$
-\frac{H}{k_{\mathrm{B}} T}=\sum_{[i, j]}\left\{K_{2}\left(s_{i} s_{j}+\sigma_{i} \sigma_{j}\right)+K_{4} s_{i} \sigma_{i} s_{j} \sigma_{j}\right\},
$$

where $K_{i}=-J_{i} / k_{\mathrm{B}} T$, with $i=2$ or $4,[i, j]$ denotes summation over nearest-neighboring lattice sites, and $T$ is the temperature of the system.

We consider this standard AT model in 3D put on the cubic lattice. It should be called the standard one as there are many extensions of the AT model (see e.g. [3]). Being non-trivial generalization of widely applied Ising model, the AT model is one of the most important reference points in statistical physics [4]. It raises current interest. Every year a dozen papers are devoted to it [3].

The state of research performed for this model and its applications are available in many papers, e.g. [5-10]. Its $K_{2}\left(K_{4}\right)$ phase diagram high complexity is the consequence of the fact that three components of the order parameter can order independently: not only $\langle s\rangle$ and $\langle\sigma\rangle$, but also $\langle s \sigma\rangle$ where $\langle\ldots\rangle$ denotes the thermal average.

The aim of our paper is to present the method of computation of latent heat based on the energy distribution histogram, originally proposed for the $q$-state Potts

\footnotetext{
*corresponding author; e-mail: djeziorek@wp.pl
}

model [11], applied by us to the 3D AT model. To confirm the applicability of this method, we compare results of our analysis with the ones obtained by us using the analysis of the behavior of the cumulants of the type of Challa $V_{L}$ [12] and Lee-Kosterlitz $U_{L}$ [11], modified by us $[7,10]$. The latter for the first time applied by us to the AT model what is explained in Section 2. Similarly as in the original method for the $q$-state Potts model [11] for strong first order phase transitions, in Section 3 we demonstrate the characteristic histogram with two peaks in the critical region also in the 3D AT model and we compute the latent heat on this basis.

\section{The method of computation}

Just like in the $q$-state Potts model with $q$ equivalent ordered states and one disordered, we observe characteristic histogram of two peaks in the critical area also in the 3D AT model. As shown on the right in Fig. 1 for spins $s$, maxima of these peaks appear at the energy value $E_{-, L}$ for the ordered state and at $E_{+, L}$ for the disordered one, and they are separated by a minimum of $E_{m, L}$. In this paper we use energy per lattice site in $k_{\mathrm{B}} T$ units.

Hamiltonian (1) is the sum of three terms, each of which shows the interaction energy within one component of the order parameter. We perform the analysis of the entire energy of the system, but it does not give an insight into the individual behavior of the degrees of freedom $s, \sigma$ and $s \sigma$ which demonstrate independent ordering. To calculate the individual contribution of each of these three degrees of freedom to latent heat, we also analyze the behavior of the energy of each degree of freedom, i.e. the mean value of each of the three terms in Hamiltonian (1) separately.

Technically, we compute the histogram dividing the whole interval of the energy $E$ (in $k_{\mathrm{B}} T$ units) occurring in our computer experiments into small subintervals and the program counts the energy appearing in each subinterval separately. Then we calculate the probability value $P$ of the energy in the different subintervals in a system 


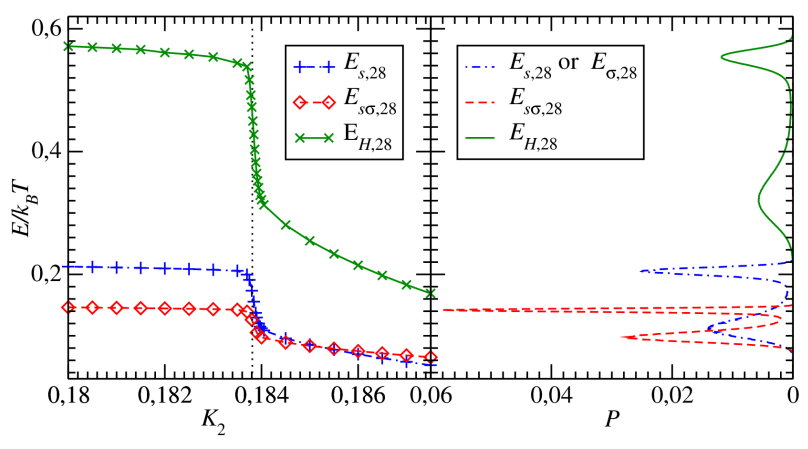

Fig. 1. On the left, the energy dependence $E_{H, 28}\left(K_{2}\right)$ of the whole system as well as the energy $E_{\alpha, 28}\left(K_{2}\right)$ of interaction of degrees of freedom $\alpha=s$ (or $\sigma$ ) or $s \sigma$ in $k_{\mathrm{B}} T$ units for the 3D AT model with $L=28$ for $K_{4}=0.1$. The critical value $K_{2}=0.18381(2)$ is marked with a vertical dotted line. On the right side is the energy distribution histogram, i.e. probability $P_{L}$ as a function of the energies specified in the legend box. For clarity only selected points are marked.

of a finite size $L \times L \times L$. Our program independently calculates the values $P_{L}\left(E_{\alpha}, \beta\right)$ and $-\ln P_{L}\left(E_{\alpha}, \beta\right)$, where $\alpha=s, \sigma, s \sigma, \beta=1 / k_{\mathrm{B}} T$ and, obviously, we also take the whole Hamiltonian (1) as argument at the $K_{2}$ critical value. This $K_{2}$ value was carefully determined from analyzes using the $Q_{L}=\left\langle\alpha^{2}\right\rangle_{L}^{2} /\left\langle\alpha^{4}\right\rangle_{L}$ [13], $V_{L}=$ $\left\langle E_{\alpha}^{4}\right\rangle_{L} /\left(3\left\langle E_{\alpha}^{2}\right\rangle_{L}^{2}\right)[12]$ and $U_{L}=\left\langle E_{\alpha}^{2}\right\rangle_{L} /\left\langle E_{\alpha}\right\rangle_{L}^{2}$ [11] like cumulants, modified by us for the AT model [7, 9, 10], for the fixed $K_{4}$ value. To compute the values of the Binder type cumulants, we use the $n$-th power of the $\alpha$ spins order parameter $\left\langle\alpha^{n}\right\rangle_{L}$. Whereas, for the Challa and the Lee-Kosterlitz like cumulants we exploit the $n$-th moment of the $\alpha$-spins order parameter interaction energy $\left\langle E_{\alpha}^{n}\right\rangle_{L}$. Moments in all cumulants are averaged over an ensemble of independent samples of the size $L \times L \times L$. Here $\alpha=s$, $\sigma, s \sigma$. For the Challa and Lee-Kosterlitz like cumulants as an argument, we also take the average energy value for the entire Hamiltonian (i.e. internal energy of the system).

Besides validating the code of computer experiment, our analyses also allow the important test: the respective energy values $E_{-}$and $E_{+}$for the ordered and unordered states defined within the individual degrees of freedom and for the entire Hamiltonian $H$ should fall in the upper and lower regions of the characteristic sudden jump of the average values of the respective energies $E_{\alpha}$ in the $K_{2}$ function at the fixed value of $K_{4}$ parameter.

For our analysis we use the property that the positions of the above-mentioned minima $E_{-, L}^{\min }$ and $E_{+, L}^{\mathrm{min}}$ of $-\ln P_{L}\left(E_{\alpha}, \beta\right)$ satisfy the relation (see Eq. (2.4) in [11])

$$
E_{i, L}^{\min }-E_{i}=O\left(L^{-(d-1)}\right),
$$

where $i=-$ or + . It is noteworthy that relation (2) has been derived under the assumption that the phase transition of the first order is strong [11]. Thus, for finite systems with ever greater dimensions $L$ the energy values $E_{i, L}^{\min }$ linearly approach their bulk values $E_{i}$ as a function of $L^{-(d-1)}$. Here $d$ is the dimensionality of the system.
In systems with one independent component of the order parameter, like the Ising or the $q$-state Potts model, dependence (2) was used as a finite-size scaling relation (see e.g. [11] and the papers cited therein). In these papers, positions of the minima $E_{i, L}^{\min }$ of $-\ln P_{L}(E, \beta)$ obtained for finite samples with $d=2$, then in the function $L^{-1}$ show good linear scalability to their thermodynamic limits to $E_{i}$ values known from the analytical calculations. Based on these results we can calculate directly the latent heat $l$

$$
l=E_{+}-E_{-} .
$$

\section{The results and conclusions}

Similarly as in the $q$-state Potts model [11], Fig. 1 on the right shows the characteristic histogram with two peaks in the critical region obtained by us for the 3D AT model of size $L=28$ at $K_{4}=0.1$. Also in this model, the probability peaks $P_{L}$ fall into the upper and lower regions of the characteristic sudden jump average value of $E_{H, 28}$ of the entire Hamiltonian, as well as the energy $E_{\alpha, 28}$ of degrees of freedom $\alpha=s$ (the same dependence for degrees $\sigma$ because of Hamiltonian (1) symmetry) and $s \sigma$ as a function of $K_{2} . K_{2}=0.18381(2)$ marked by the vertical dotted line in Fig. 1 has been carefully determined by us as a critical value based on the analyses of the behavior of the above mentioned Binder [13], Challa [12] and Lee-Kosterlitz [11] like cumulants and has the same value for each of the three degrees of freedom of the system. Locating accurately the phase transition point is crucial for this method [11].

Of course at every abscissa on the left in Fig. 1 the sum of the mean values of the respective interaction energy of degrees of freedom $s, \sigma$ and $s \sigma$ equals to the mean value of the internal energy of the whole system within the uncertainty intervals that are of the order of the symbol magnitude. This is the next positive test for validity of the code of our computer program. It is worth pointing out that our results refer to the boundary between the Baxter phase, where all the components of the order parameter $\langle s\rangle,\langle\sigma\rangle$ and $\langle s \sigma\rangle$ are ordered, and the paramagnetic one.

As explained in Sect. 2, our program computes $-\ln P_{L}$ as a function of energy $E_{\alpha, L}$ not only of the whole Hamiltonian, but also of the energy $E_{\alpha, L}$ of interaction of degrees of freedom $\alpha=s, \sigma$ and $s \sigma$ separately for a system of different $L$ sizes. Figure 2 shows this dependence $-\ln P_{L}\left(E_{\alpha, L}\right)$ for degrees of freedom $s$ (or $\sigma$ ) with $K_{4}=0.1$ at critical value $K_{2}=0.18381(2)$ for system size values $18 \leqslant L \leqslant 32$ explained in the legend box.

Based on these graphs, we determined the positions of both minima occurring in the relation (2), which is the most probable energy of $E_{-, L}$ for ordered state (left minima) and of $E_{+, L}$ dominating for unordered one (right minima). In order to increase the precision of minima position determination, in their vicinity a smooth approximating curve is used, applying regression of the third degree. The approximated curves are illustrated by the solid lines in Fig. 2. 


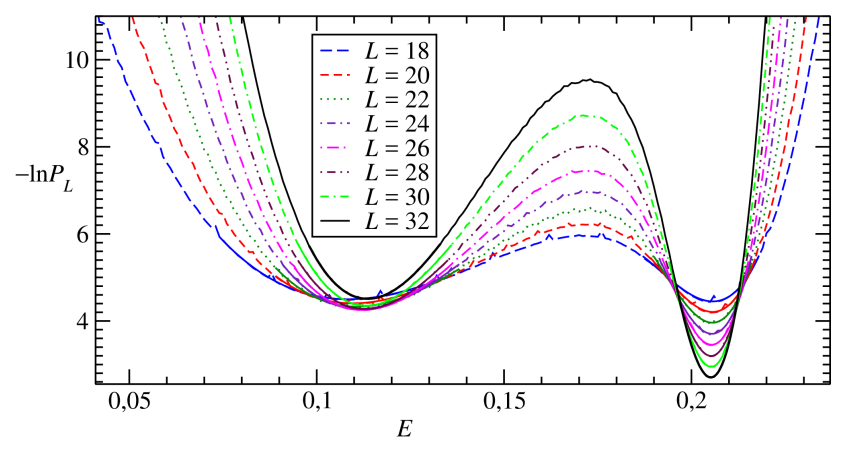

Fig. 2. The course of dependence $-\ln P_{L}\left(E_{\alpha}, \beta\right)$ for degrees of freedom $s$ (or $\sigma$ ) at the critical value of $K_{2}=$ $0.18381(2)$ and $K_{4}=0.1$. The values of the system size $L$ are explained in the legend box. In the minima regions the dependencies $-\ln P_{L}\left(E_{\alpha}, \beta\right)$ have been smoothed by the solid curves using the third degree regression.

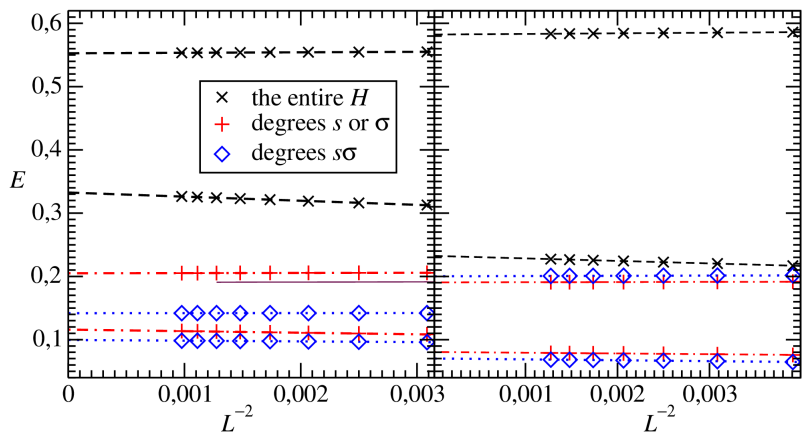

Fig. 3. Values of $E_{+, L}^{\mathrm{min}}$ (upper lines) and $E_{-, L}^{\mathrm{min}}$ (lower lines) of the minima positions of dependencies $-\ln P_{L}\left(E_{\alpha}, \beta\right)$ received at $K_{4}=0.1$ (left) and $K_{4}=0.17$ (right) for a system of finite size $L$ for degrees of freedom explained in the legend box. The individual lines are extrapolated to their thermodynamic limits $E_{+}$or $E_{-}$using linear regression.

Symbols in Fig. 3 illustrate the results of such analyzes for energies $E_{+, L}^{\mathrm{min}}$ and $E_{-, L}^{\mathrm{min}}$ for the whole Hamiltonian (1), as well as for the energy of interaction of degrees of freedom $s, \sigma$ and $s \sigma$ separately, explained in the legend box, at $K_{4}=0.1$ (left side) and at $K_{4}=0.17$ (right side). According to the formula (2), the values of $E_{+, L}^{\min }$ and $E_{-, L}^{\min }$ in the $L^{-2}$ function scale to the respective bulk values $E_{+}$and $E_{-}$. Therefore, the individual lines in Fig. 3 according to the relation (2) are extrapolated to the thermodynamic limit using linear regression.

The resulting values $E_{+}$and $E_{-}$after substituting into equation (3) at $K_{4}=0.1$ give latent heat $l_{s}=0.0891(5)$ for spins $s$ (or $\sigma$ ) and $l_{s \sigma}=0.0423(6)$ for degrees of free$\operatorname{dom} s \sigma$, which sum up to $l_{\text {sum }}=0.2205(16)$. While latent heat determined on the basis of the entire internal energy is $l_{H}=0.2202(9)$. At $K_{4}=0.17$ we have obtained $l_{s}=0.1100(4), l_{s \sigma}=0.1300(4), l_{\text {sum }}=0.3500(12)$, and $l_{H}=0.3499(6)$. Results $l_{H}$ and $l_{\text {sum }}$ are consistent within the uncertainty intervals.
Thus, the method of computation of latent heat based on the energy distribution histogram can successfully be used in systems with many components of an order parameter showing individual ordering. The latent heat values received here are consistent within the uncertainty intervals with the values obtained from the analysis of the behavior of the cumulants of the type of Challa $V_{L}$ [12] $l=0.2197(14)$ at $K_{4}=0.1$ and $l=0.3504(23)$ at $K_{4}=$ 0.17 as well as of Lee-Kosterlitz $U_{L}[11] l=0.223(15)$ at $K_{4}=0.1$, modified by us $[7,10]$.

This method requires much less numerical computation, and the results for extrapolation to the thermodynamic limit in Fig. 3 exhibit significantly lower statistical scattering than those based on the Challa and LeeKosterlitz cumulants. However, in order to use it, it is necessary to have quite precisely determined position of the investigated phase transition.

\section{Acknowledgments}

The authors wish to thank the Poznań Supercomputing and Networking Center as well as Faculty of Physics at Adam Mickiewicz University for the access to the computing platforms on which computations have been performed. Separate thanksgiving are for Professors G. Kamieniarz and W. Florek for valuable discussions.

\section{References}

[1] J. Ashkin, E. Teller, Phys. Rev. 64, 178 (1943).

[2] C. Fan, Phys. Lett. 39A, 136 (1972).

[3] J. P. Santos, F. C. Sá Barreto, Braz. J. Phys. 46, 70 (2016).

[4] R. J. Baxter, Exactly Solvable Models in Statistical Mechanics, Academic Press, London 1982.

[5] P. Arnold, Y. Zhang, Nuclear Phys. B 501, 803 (1997).

[6] L. F. Feiner, A. M. Oleś, Phys. Rev. B 59, 3295 (1999).

[7] G. Musial, Phys. Rev. B 69, 024407 (2004).

[8] G. Szukowski, G. Kamieniarz, G. Musiał, Phys. Rev. E 77, 031124 (2008).

[9] D. Jeziorek-Knioła, G. Musiał, L. Dębski, J. Rogiers, S. Dylak, Acta Phys. Pol. A 121, 1105 (2012).

[10] D. Jeziorek-Knioła, G. Musiał, Z. Wojtkowiak, Acta Phys. Pol. A 127, 327 (2015).

[11] J. Lee, J. M. Kosterlitz, Phys. Rev. B 43, 3265 (1991).

[12] M.S.S. Challa, D.P. Landau, K. Binder, Phys. Rev. B 34, 1841 (1986).

[13] K. Binder, D.P. Landau, Phys. Rev. B 30, 1477 (1984). 